\title{
Dimensionen eines gesellschaftspolitischen Kurswechsels - Überlegungen zu gewerkschaftlichen Zielen und ihren Durchsetzungsstrategien
}

\section{Die Krise als Herausforde- rung zum Pfadwechsel}

Die Krise der Weltwirtschaft, die Ende 2008 eingetreten ist, stellt eine Zäsur dar. Sie zeigte sich schon von Anbeginn an als die schwerste globale Krise des Kapitalismus seit der Großen Depression in den 1930er Jahren. Deutschland als Exportnation war besonders betroffen. Fast alle Industriebetriebe mussten zweistellige Produktionsrückgänge hinnehmen, manche waren in ihrer Existenz bedroht. Das Bruttoinlandsprodukt (BIP) in Deutschland sank um fast $5 \%$ - ein in der Geschichte der Bundesrepublik beispielloser Einbruch.

Die deutschen Gewerkschaften haben gerade 2009 einen wichtigen Beitrag zur erfolgreichen Krisenintervention geleistet. Das Ziel der IG Metall und ihrer Betriebsräte war es, in dieser zugespitzten Situation Massenentlassungen zu verhindern, Arbeitsplätze und Betriebe zu erhalten. Die etablierten betrieblichen und überbetrieblichen Aushandlungsstrukturen waren eine notwendige Voraussetzung zur schnellen und pragmatischen Um- und Durchsetzung vieler Kriseninterventionsinstrumente wie der verlängerten Kurzarbeit oder der kollektiven Nutzung der Arbeitszeitkonten.

Einige der Maßnahmen - etwa die Konjunkturpakete, die Umweltprämie für Altautos und die Kurzarbeitsregelung - können als Rückbesinnung auf keynesianische Krisenbekämpfungsstrategien ge- sehen werden. Offensichtlich war die Ratlosigkeit im wirtschaftswissenschaftlichen und politischen Mainstream so groß, dass für die kurzfristige Krisenbekämpfung die über viele Jahre verteufelten Instrumente keynesianischer Politik wieder hoffähig wurden.

Angesichts einer schnelleren wirtschaftlichen Erholung, als die meisten dies erwartet hatten, gab es in Teilen der politischen Linken eine Debatte darüber, ob das Zeitfenster für grundlegende Reformen bereits wieder geschlossen wäre und sich das erschütterte Weltbild des Marktradikalismus wieder rekonstituiert hätte. Diese Debatte verkannte, dass die zentralen Krisenursachen nicht beseitigt waren und die Politik des „Weiter so“" schneller wieder in Legitimationskrisen geraten musste als von vielen gedacht.

Die Gefahr einer sogenannten Double-Dip-Rezession, einer Wiederkehr der Rezessionsgefahr nach einer kurzfristigen sprunghaften Erholung, blieb real, weil die Regierungen Europas auf die Verschuldungskrise in einigen europäischen Ländern - die zu erheblichen Teilen auf die notwendigen staatlichen Interventionen in den Jahren 2008/09 (Bankenrettung etc.) zurückzuführen sind - mit rigiden Spardiktaten reagierten (und weiterhin reagieren), die die Konjunktur abwürgten und so die Verschuldungsproblematik der öffentlichen Haushalte noch verschärften. Damit wiederholte sich eine Situation, die aus der Weltwirtschaftskrise der 1930er Jahre schon bekannt war. Auch damals hatten Regierungen, wie z.B. die der Vereinigten Staaten, nach einer kurzen konjunkturellen Erholung die nachfrage- stimulierenden Programme und Strukturreformen heruntergefahren und auf die angestiegene Staatsverschuldung mit einer Austeritätspolitik reagiert - und gerade dadurch den zweiten schwereren konjunkturellen Absturz im Jahr 1937 ausgelöst. Erst durch diesen Schock - und nicht durch die 1936 erschienene „General Theory“ von John Maynard Keynes - wurde die Einsicht unabweisbar, dass es sich nicht um eine vorübergehende, konjunkturelle Krise, sondern um eine tief greifende Strukturkrise des Kapitalismus handelte, die auch nur durch grundlegende nationale und internationale Strukturreformen des kapitalistischen Wirtschafts- und Gesellschaftsmodells bewältigt werden konnte.

Eine sehr ähnliche Situation trat im Sommer 2011 mit der Verschuldungs- und Eurokrise ein. Die durch die Politiken des „Weiter so" ausgelöste Rezessionsgefahr wurde für große Teile des langfristig denkenden liberalen und konservativen Lagers der zweite Schock, der ein Umdenken einleiten könnte. Unter dem Titel „How to avoid a double dip“

Martin Allespach, Dr. habil., Leiter
des Bereichs Grundsatzfragen und
Gesellschaftspolitik beim Vorstand der IG
Metall und Privatdozent an der Universität
Hamburg.
e-mail: Martin.Allespach@igmetall.de
Martin Bartmann, M.A., Mitarbeiter
im Bereich Grundsatzfragen und
Gesellschaftspolitik beim Vorstand der
IG Metall und Lehrbeauftragter an der
Universität Mainz.
e-mail: Martin.Bartmann@igmetall.de


forderte schon Ende August der liberale Economist in erstaunlicher Klarheit einen Kurswechsel und eine Abkehr von der Austeritätspolitik in Europa: „Im Jahr 2008 wurde die Weltwirtschaft durch einen mutigen und koordinierten Plan, die Banken zu stützen und dem Abschwung mit einem fiskalischen und monetären Stimulus zu begegnen, gestützt. Heute fehlt ein solcher Mut (die Krise der Euro-Zone steht für Politiker, die zu wenig zu spät tun). Es gibt keine Koordination. [...] Im Geist von 2008 könnten die policy makers eine koordinierte Strategie einschlagen, um dem Wachstum Auftrieb zu geben. [...] Die Erholung wäre dann immer noch unsicher und langsam. Aber sie hätte eine weit bessere Chance, sowohl eine Rezession als auch eine Stagnation zu verhindern als das der heutige policy mix tut. Es ist höchste Zeit, den Kurs zu wechseln." (The Economist vom 27.8.2011, S. 10).

Seit dieser Mahnung hat sich die Krise Woche um Woche weiter gesteigert, zunächst als Euro-Krise, aber verbunden mit der zunehmenden Sorge um eine weltweite Kettenreaktion und unter der Begleitmusik vielfältiger neuer Protestbewegungen, die die Kontrolle des spekulativen Finanzkapitals und die Wiederherstellung des Wohlfahrtsstaates, den Abbau sozialer Ungerechtigkeit und die erhöhte demokratische Mitwirkung der Bürger fordern.

An diesem Aufbruch zu einem Kurswechsel sind in vielen Ländern auch die Gewerkschaften beteiligt. Die IG Metall, die ihn schon lange zu einem zentralen Thema gemacht hatte (vgl. Huber 2010), hat in der Programmatik ihres Gewerkschaftstages im Oktober $2011^{1}$ die Notwendigkeit eines in langfristigen, gesamtwirtschaftlichen, europäischen und globalen Strategien und Zielen verankerten Kurswechsels bekräftigt und sich als ein zentraler gesellschaftspolitischer Akteur in der Debatte um die notwendigen Richtungsänderungen zu Wort gemeldet. In ihrer einstimmigen „Karlsruher Erklärung“ bekannte sie sich $\mathrm{zu}$ einer europäisch-solidarischen Krisenlösung, die auf einem „entschiedenen und sofortigen Kurswechsel in der Finanz-, Wirtschafts- und Arbeitsmarktpolitik der EU-Kommission und der deutschen Bundesregierung hin zu einem demokratischen, ökologischen und sozialen Europa" beruht (IG Metall 2011).

Doch der Prozess des Umsteuerns bewegt sich insgesamt nur zögerlich und kommt allein durch den immer wieder erneuerten Druck der Umstände in kleinen
Schritten voran. Die Hegemonie der neoliberalen Politik ist keineswegs gebrochen. Negativ-Beispiele für staatliche Mittelvergeudung und Ineffizienzen - wie sie in einigen der besonders betroffenen Länder heute nicht zu leugnen sind - werden in den Vordergrund gestellt, um den riesigen Bedarf an öffentlichen Investitionen in Infrastrukturen, Bildung und Forschung, Gesundheit, soziale Sicherheit und nicht zuletzt in ökologische Technologien und Produkte zu verdecken und zu ignorieren.

Doch es zeigen sich zunehmend Risse in den neoliberalen Interessenkoalitionen. Auch in Deutschland rücken wichtige konservative Vordenker zunehmend von dieser Interessenkoalition ab. In Anlehnung an den konservativen britischen Publizisten Charles Moore beschrieb der FAZHerausgeber Frank Schirrmacher Mitte August 2011 unter dem Titel „Ich beginne zu glauben, dass die Linke recht hat" die tiefe Verunsicherung über die Kaperung und den Missbrauch bürgerlicher Werte durch den Neoliberalismus (Schirrmacher 2011). Das Zeitfenster für Reformen ist offensichtlich nicht geschlossen, auch wenn die Schwierigkeiten der Umsetzung eines Pfadwechsels unverkennbar sind. Er wird freilich nicht von selbst kommen, sondern gesellschaftspolitische Akteure, wie bspw. die Gewerkschaften, müssen ihn aktiv skizzieren und dazu beitragen, ihn mehrheitsfähig zu machen.

\subsection{WOHLFAHRTSSTAATLICHE PFADE ALS KRISENBEWÄLTIGUNG}

Dabei ist es wichtig, einen Blick zurück auf die klassischen Pfade des politisch regulierten Kapitalismus zu werfen, insbesondere auf die verschiedenen Modelle des kapitalistischen Wohlfahrtsstaates, die durch die noch von Keynes mit beeinflusste internationale Ordnung des Finanzsystems flankiert waren. Fraglos können diese Modelle unter den heutigen weiterentwickelten weltwirtschaftlichen und weltpolitischen Bedingungen nicht einfach retrospektiv wiederbelebt oder gar implementiert werden. Sie bleiben aber eine Bezugsgröße und ein Lehrstück dadurch, dass auch damals bestimmte Akteursgruppen sich zu Koalitionen zusammengefunden haben, um die Weltwirtschaftskrise der 1930er Jahre zu bewältigen.

Im historischen Rückblick auf den New Deal in den Vereinigten Staaten wird ein auch heute relevantes Handlungsmuster sichtbar. Wie schon C. Wright Mills (1948) in seiner klassischen Studie über die Gewerkschaften als neue Akteure der Gesellschaftspolitik beobachtet hat, standen sich in der damaligen Weltwirtschaftskrise Kapital und Arbeit nicht als geschlossene Gruppen gegenüber, sondern geteilt in kurzfristig und langfristig denkende „Fraktionen“. Nach und nach setzten die langfristig denkenden Fraktionen beider Seiten einen neuen Interessenkompromiss durch, den korporativen, von den großen Kapitalgesellschaften und Industriegewerkschaften getragenen Konsensus über ein ausgleichendes Sozialmodell, in dem die Kaufkraft der Arbeitnehmer und die sozialen Staatsausgaben wachsen sollten.

Zustande gekommen war die Interessenkoalition des „New Industrial State“ (Galbraith 1967) nicht allein aus keynesianischer Einsicht, sondern auch durch den Konflikt zwischen den großen Kapitalgesellschaften, die auf langfristige Plan- und Kalkulierbarkeit angewiesen waren, und dem spekulativen Aktionärskapital, dessen Lust am kurzfristigen Risiko in den Börsenkrach von 1929 geführt hatte. Der neue Wohlfahrtsstaat stabilisierte nicht nur den Kapitalismus mit seinen Risiken und Konflikten. Er erweiterte zugleich auch die gewerkschaftliche Gegenmacht, die Rechte der Arbeitnehmer auf Schutz und auf Mitbestimmung und die allgemeinen Standards des Gesundheits- und Bildungswesens und der öffentlichen Infrastrukturen.

Allerdings unterschied sich die Teilhabe der sozialen Gruppen am Wohlfahrtsstaat nach den nationalen Machtverhältnissen und „Entwicklungspfaden“ (Esping-Andersen 1998). Am größten war sie im sozialdemokratisch-skandinavischen, geringer im konservativ-kontinentaleuropäischen und am geringsten im angelsächsischliberalen Wohlfahrtsstaat. Gleichwohl erkämpften die Gewerkschaften in den Wachstumsjahren bis nach 1970 überall ein Aufrücken der arbeitenden Klasse in Standards der sozialen Mitte und in vielen Ländern, insbesondere der BRD, auch eine nicht geringe demokratische Mitbestimmung in den Betrieben und der Gesellschaftspolitik.

Die von Gøsta Esping-Andersen mit seinem Buch „The Three Worlds of Welfare

1 Der Gewerkschaftstag der IG Metall stand unter dem Motto: "Kurswechsel: Gemeinsam für ein Gutes Leben". 
Capitalism" angestoßene neuere Debatte über die verschiedenen Pfade der „politischen Ökonomien des Wohlfahrtsstaates" (Esping-Andersen 1990, S. 9) macht die Komplexität der Bedingungen und Kräftefelder deutlich, aus denen Pfade und Pfadänderungen zustande kommen (vgl. hierzu Hübner und Bogedan in diesem Heft). Esping-Andersen hatte insbesondere die zuerst von Theodor Geiger (1949) entwickelte Theorie der politischen Intervention in die Klassenbeziehungen (vgl. hierzu Weber-Menges/Vester in diesem Heft) wiederbelebt.

Nach seiner „institutionellen Schichtungstheorie" (Esping-Andersen 1993, S. 2) hängen die Klassenverhältnisse und Proportionen der gesellschaftlich-beruflichen Arbeitsteilung nicht allein von den „nackten“ ökonomischen Marktinteressen ab. Sie sind auch nicht auf funktional determinierte Antworten auf Industrialisierungs- oder Modernisierungsgrade oder auf strukturelle Komponenten (z.B. die demografische Entwicklung) zu beschränken. Sein in deutscher Sprache erschienener historischer Abriss der Pfade (Esping-Andersen 1998) macht nicht zuletzt deutlich, dass Pfade und Pfadwechsel in aller Regel nicht durch einzelne Akteure zustande kommen, sondern durch das gesamte Kräftefeld der Akteure und Institutionen; sie folgen keinem von oben durchsetzbaren „Masterplan“, sondern sind das Ergebnis einer Abfolge verschiedenster Konflikte und Kompromissbildungen. Sie sind von den historischen Kämpfen und Kompromissen der großen gesellschaftlichen Gruppen geprägt, deren Grundmuster oft weit zurück in die nationale Geschichte reichen. Die Analyse solcher Konstellationen kann nicht in der isolierten Betrachtung der einem Akteur zur Verfügung stehenden Machtressourcen (z.B. des Anteils der Parlamentssitze einer Partei, der Organisationsgrade von Gewerkschaften und Arbeitgeberverbänden) beurteilt werden, sondern es müssen sowohl die Stärke und Kohäsion konkurrierender Akteure und die Dauerhaftigkeit ihrer Mobilisierungskraft als auch die Fähigkeit zur Bildung von Machtallianzen berücksichtigt werden. ${ }^{2}$

\subsection{KONSERVATIVE, SOZIALDEMO- KRATISCHE UND LIBERALE PFADE}

Die historischen „Pfade“ sind nach EspingAndersen auf vier Handlungsebenen recht- lich und institutionell verfestigt: (1) in den Institutionen des Staates, (2) im tarifpolitischen Konflikt- und Aushandlungssystem, (3) im Modell der Familie und der geschlechtlichen Arbeitsteilung und (4) in den Teilhabe- und Mitwirkungsrechten der Bürgerinnen und Bürger (vgl. auch Marshall 1989). Sie unterscheiden sich danach, ob ihre Politiken eine polarisierte, eine in der Mitte integrierte oder eine hierarchisch gestufte Gesellschaftsordnung begünstigen. Das heißt, die Forderungen und Intentionen der relevanten politischen Akteure lassen sich dem Liberalismus, dem demokratischen Sozialismus und dem Konservatismus zuordnen. Aus diesen drei politischen Traditionen bzw. aus den aus ihnen entstandenen normativen gesellschaftspolitischen Konzeptionen (Kohl 1993, S. 68) entwickelt Esping-Andersen seine Wohlfahrtstaatstypologie, in der er ein liberales, ein konservatives und ein sozialdemokratisches Wohlfahrtsstaatsmodell unterscheidet. ${ }^{3}$

Der sozialdemokratische Pfad, der aus den vergleichsweise egalitären Traditionen Skandinaviens entstand, erstrebt insbesondere die Anhebung der unteren Schichten auf die individuellen Lebenschancen der modernen Mittelschichten und die Gleichstellung der Frauen. Es bietet eine steuerfinanzierte staatliche Vorsorge und, als Alternative zu Prekarität oder Erwerbslosigkeit, mehr Arbeitsplätze auf den mittleren Rangstufen der Gesundheits-, Bildungs- und Sozialdienstleistungen. Das Modell ist geprägt durch einen stark investiven Wohlfahrtsstaat (Schwerpunkte auf aktiver Arbeitsmarktpolitik und hohen Bildungsinvestitionen). Es setzt auf Wachstum, relativ geringe Ungleichheiten und strebt eine Kopplung aus sozialer Befriedung und wirtschaftlicher Effizienz an.

Der konservative Pfad geht auf die korporativen Traditionen des kontinentalen Westeuropa zurück, die sich vom Laissez-faire-Kapitalismus abgrenzen. In Deutschland wurden die Grundzüge dieses Modells vor allem im Klassenkompromiss des Bismarckschen „Sozialversicherungsstaates" institutionalisiert. Es beruht auf einem Arrangement zwischen ständischen Interessen (Besitzstands- und Statussicherung), marktwirtschaftlichen Interessen (Leistungsdifferenzierung) und sozialintegrativen Interessen (sozialer Ausgleich). Familienpolitisch basiert es auf dem patriarchalischen Alleinverdienermodell. Die soziale Ungleichheit wird abgefedert durch die Sicherung des bisherigen Platzes (der Gesellschafts-, Geschlechts- und Altersklassen) in einer Hierarchie gestufter Rechte und Pflichten.

Dass im deutschen Wohlfahrtsstaat die Sicherung einer großen arbeitnehmerischen Mitte erfolgreich war, ist durch das „industrielle Produktionsmodell“ ermöglicht worden, das die internationale Vorrangstellung der deutschen Exportwirtschaft begründet hat. Sie beruhte auf einer hochwertigen Produktionsleistung, allerdings mit Fachkräften, deren hohe Arbeitsleistung vor allem auf der mittleren Ebene der Berufs- und Allgemeinbildung eingestuft und bezahlt wurde. Nicht wenigen Arbeitnehmern konnte, allerdings seit den 1990er Jahre in deutlich abnehmendem Umfang, der begrenzte Aufstieg durch gute und sichere Arbeitsplätze, die mit mittlerer Bildung erreicht werden konnten (Müller 1998), schmackhaft gemacht werden.

Der liberale Pfad der angelsächsischen Länder knüpft an die radikale liberale Laissez-faire Politik an. Diese hatte die starken sozialen Polarisierungen und Konfrontationen während der industriellen Revolution herbeigeführt. Die neoliberale Neuauflage des Laissez-faire verstärkte nun, durch Deregulierung und staatliche Sparpolitik, vertikale Polarisierungen. Im Interesse der bürgerlichen Mittel- und Oberschichten wird auf individuelle Konkurrenz um sozialen Aufstieg und Abstieg gesetzt, deren Risiken nicht von der Gesellschaft, sondern durch private Selbstvorsorge abgesichert werden sollen. Diejenigen, die nicht mithalten können, werden auf staatliche Minimalsicherungen bzw. Beschäftigungen im expandierenden Niedriglohnsektor verwiesen.

\footnotetext{
2 Die Pfaddebatte und die aus ihr entstandenen Typologien begrenzen sich in der Regel auf Unterschiede innerhalb des Kapitalismus. Staatssozialistische Systeme stehen nicht im Fokus. Ihre Existenz spielt allenfalls als Rahmenbedingung (Drohung der Systemalternative) für die Akteure in den untersuchten kapitalistischen Ländern eine Rolle.

3 Diese drei Wohlfahrtsstaatstypen können als Idealtypen im Sinne Max Webers begriffen werden (vgl. Kohl 1993, S. 69). Sie sind aus den unterschiedlichen gesellschaftspolitischen Konzeptionen theoretisch entwickelt und der Durchdringungsgrad der realen Wohlfahrtsstaaten - die in der Regel ein Mix aus Elementen der Idealtypen darstellen - wird an ihnen "gemessen“. Gleichwohl handelt es sich nicht um ein statisches Konzept, da sich auch die gesellschaftspolitischen Konzeptionen weiter entwickeln, sodass neue Herausforderungen -z.B. Ökologie und Klimawandel - neue Antworten und Konzepte erfordern.
} 
In der weiteren Diskussion beschäftigt sich Esping-Andersen mit den Herausforderungen, denen die Pfadkonzepte durch die internationale Deregulierung der Weltwirtschaft zunehmend ausgesetzt waren. In dem Sammelband „Why We Need a New Welfare State" wird deutlich, dass er eine Chance sieht, diese Herausforderungen zu bewältigen, in einer Art Konvergenz, in der die Modelle jeweils Elemente der anderen Modelle übernehmen (Esping-Andersen et al. 2002). Diese Diskussion wurde jedoch nicht fortgesetzt, obwohl sie immer notwendiger wurde - nicht zuletzt in der Auseinandersetzung mit der 2003 durchgesetzten „Agenda 2010“ der rot-grünen Koalition in Berlin. Es folgten kaum weitere Beiträge von Esping-Andersen zu der Thematik, und die von ihm und um ihn initiierte soziologische Pfaddiskussion der Soziologen versandete zunächst.

Fortgesetzt worden ist die Pfaddiskussion vor allem unter politikwissenschaftlichen Aspekten von Wissenschaftlern wie Wolfgang Streeck und Wolfgang Merkel. Wiederbelebt wird sie seit Kurzem von einer Reihe von jüngeren Wissenschaftlerinnen und Wissenschaftlern, die die Pfadentwicklungen in vergleichenden Länderstudien untersuchen. ${ }^{4}$

In der neueren Literatur zur Pfadentwicklung werden Pfadwechsel beschrieben, die sich erst aus mehreren, durchaus auch über längere Zeiträume sich verstärkenden „kritischen Entscheidungen“ entwickeln. Pfadwechsel müssen nach Merkel nicht aus einer großen Entscheidung, einem Wendepunkt entstehen, „sondern können auch in einer zeitlich begrenzten Sequenz sich verstärkender Entscheidungen bestehen.“ (Merkel 2010, S. 73)

\subsection{SOZIALE UND ÖKOLOGISCHE ENTWICKLUNG DES BESCHÄFTI- GUNGSSYSTEMS}

Ein zweiter wissenschaftlicher Impuls zur Erneuerung der Pfaddiskussion kommt von der fortgeschrittenen keynesianischen Makroökonomie, deren Diskussion insbesondere von den Nobelpreisträgern Paul Krugman und Joseph Stiglitz organisiert und repräsentiert wird. Durch ein sorgfältiges Studium der Wirtschaftsgeschichte und nationale Fallstudien seit der großen Depression arbeiten sie die risikobehafteten Logiken der Entwicklungen eines Kapitalismus heraus, der vom deregulierten Finanzmarktkapitalismus dominiert ist. In
Abgrenzung vom Bild eines Vulgär-Keynesianismus, der, bildlich gesprochen, das Geld zum Fenster hinauswirft, plädieren sie für eine Wiederaufnahme einer Politik, die ein ökologisch verantwortliches Wachstum, produktive Investitionen, die Senkung der Arbeitslosigkeit und einen Ausbau des Wohlfahrtsstaats und der Bildungsinstitutionen miteinander verbindet. Was qualifizierte Beschäftigung für wachsende Zukunftsaufgaben schafft, das schafft auch Arbeitsplätze und gesellschaftliche Integration.

Damit wird die Formel eines wohlfahrtsstaatlichen Keynesianismus für veränderte historische Bedingungen weiterentwickelt, um die beiden klassischen Ziele, ökonomische Leistungsfähigkeit und soziale Gerechtigkeit, weiterhin miteinander zur Deckung zu bringen. Die Formel verkörpert die langfristige, strukturverändernde Perspektive. Als bündige Zusammenfassung ist immer noch die viel diskutierte Krisendiagnose aktuell, die Stiglitz zusammen mit dem französischen Ökonomen Jean-Paul Fitoussi und einer großen internationalen Expertengruppe schon 2009 verfasst hat (Fitoussi/Stiglitz 2009). Schon der Titel - „The Ways Out of the Crisis and the Building of a More Cohesive World“ - vereinigt in sich die beiden Ziele: das wirtschaftliche und das soziale, das die Bewältigung der ökologischen Katastrophe einschließt.

Gefordert wird in dem Gutachten eine neue Politik, „die eine Zukunft schafft, in der Wachstum nachhaltiger und umweltfreundlicher ist und in der seine Früchte auf gerechtere Weise verteilt werden“. ${ }^{5}$ Die Krise habe vor allem „strukturelle Ursachen“; sie beruhe auf einem „allgemeinen Absinken der Gesamtnachfrage" und nicht allein auf der unzureichenden Regulierung des Finanzsektors. „Die allgemeine Nachfrageschwäche ging der Finanzkrise voraus und beruhte auf strukturellen Veränderungen der Einkommensverteilung. Seit 1980 stagniert in den meisten fortgeschrittenen Ländern das mittlere Arbeitseinkommen und es haben, zum Vorteil der hohen Einkommen, die Ungleichheiten zugenommen." (ebd.)

Das Gutachten schlägt vor, „diesen Trend in der Verteilung umzukehren und dadurch zu einer mittel- und langfristig selbsttragenden Gesamtnachfrage beizutragen". Erreicht werden soll dies durch eine Erhöhung des fiskalischen Stimulus und der staatlichen Intervention, die nicht nur kurzfristige Konjunkturanregungen bringt, sondern auch mit langfristigen Struktureffekten und Entwicklungsstrategien der betreffenden Länder verbunden ist. „Infrastrukturausgaben sollten beispielsweise gegenüber laufenden Ausgaben bevorzugt werden. [...] Wünschenswert sind Investitionen in neue Umwelt- und Energietechnologien, aber auch Ausgaben, die auf den Abbau von Ungleich heit zielen oder auf die Erhöhung des Humankapitals (Bildungs- und Gesundheitsreform).“

Nachhaltiges Wachstum und höhere Niveaus der Beschäftigung sollen verbunden werden mit „einer allgemeinen Erneuerung (redesign) des Wohlfahrtssystems, die auf Umverteilung und auf die Bildung von Humankapital zielt." Dazu würden vor allem „die Verallgemeinerung einer universellen Gesundheitsvorsorge und Bildungsversorgung“" (ebd.) und eine Rückkehr zu einer soziale Unterschiede ausgleichenden Sozialversicherung gehören.

Finanziert werden soll dieser Kurswechsel zu einem ökologischen Wohlfahrtsstaat durch die Rückkehr zum Prinzip der progressiven Besteuerung insbesondere hoher und höchster Einkommen, flankiert durch eine internationale Zusammenarbeit zur „Vermeidung von Steuerwettbewerb, Lohndämpfung und Sozialdumping, der modernen Versionen der Politiken des ,Beggar-Thy-Neighbour (plündere deinen Nachbarn), die in den 1930er Jahren üblich waren." (ebd.)

Es geht also insgesamt nicht nur um „moralische Wohltaten“ und Fragen der gerechten Verwirklichungschancen für alle Bevölkerungsgruppen. Es geht vielmehr um eine langfristige Stabilisierung der Nachfrage, um die Entwicklung des „Humankapitals" und die Wiederherstellung des sozialen Zusammenhalts von Gesellschaften und um die langfristige ökologische Überlebensfähigkeit.

Das Gutachten hat die Nahtstelle zwischen Wirtschaftsentwicklung und Beschäftigungssystem benannt: den Ausbau von Arbeitsplätzen des Gesundheits-, Bildungs- und Sozialsystems, mit dem gleichzeitig auch die technische Intelli-

\footnotetext{
4 Vgl. hierzu u.a. den Beitrag von Bogedan in diesem Heft, der, wie auch der Aufsatz von Hübner in diesem Heft, das Argument der "Pfadgebundenheit “ deutlich herausarbeitet.

5 Die Quelle dieses wie auch der folgenden Zitate ist Fitoussi/Stiglitz (2009), wobei die zitierten Passagen von den Autoren dieses Beitrags übersetzt worden sind.
} 
genz, das sogenannte „Humankapital“, für eine ökologisch fortschrittliche Industrieentwicklung gefördert wird. An dieser Nahtstelle schließt der Beitrag von Vester (in diesem Heft) an, in dem eine von Vester et al. (2009) durchgeführte Studie zur Entwicklungsdynamik der Berufsstruktur vorgestellt wird. Diese Studie belegt, dass die anhaltenden Verschiebungen der Berufsstruktur nicht in eine nachindustrielle Dienstleistungsgesellschaft führen, sondern in eine industrielle Struktur auf höherer Entwicklungsstufe. In dieser drängt das Wachstum quasi von sich aus vor allem zu den von Fitoussi und Stiglitz genannten Berufsfeldern: zur Hochqualifikation unter Einschluss einer wachsenden technischen Intelligenz und zu den wohlfahrtsstaatlichen Dienstleistungen in Gesundheit, Bildung und Sozialwesen. Damit vermehren sich auch die Arbeitsplätze, auf denen der Frauenanteil zwar nicht gleich, aber doch vergleichsweise höher ist.

Aber alle diese Dynamiken sind durch die derzeitigen Pfadeigenheiten der Bundesrepublik, in denen neoliberale Elemente sich mit ständischen-konservativen und patriarchalischen Elementen mischen (vgl. Bogedan und Lehndorff in diesem Heft), geringer als in anderen hochentwickelten Ländern, insbesondere in Schweden. Eine Pfadveränderung könnte diese Dynamiken, die für die wirtschaftliche und die soziale Dynamik gleichermaßen wichtig sind, freisetzen.

\section{Politikfelder eines Kurs- wechsels und mögliche Interessenkoalitionen}

Politikfelder stehen in Wechselwirkungen zueinander. Der politische Wille zu Pfadveränderungen muss daher immer mögliche Interdependenzen im Blick haben. Wir skizzieren im Folgenden - ohne Anspruch auf Vollständigkeit - acht zentrale Politikfelder für ein sozial-ökologisches Prosperitätsmodell.

\subsection{REGULATION DER FINANZ- MÄRKTE}

In der aktuellen Eurokrise wird deutlich, dass die Finanzmärkte nach wie vor nicht unter politischer Kontrolle sind. Politische
Entscheidungen werden von den Entwicklungen an den Finanzmärkten und deren kurzfristigen Orientierungen getrieben. Aber immerhin: Es gibt durchaus Schritte in Richtung einer stärkeren Regulierung der Finanzmärkte. Selbst konservative Politiker fordern die Einführung einer Finanzmarkttransaktionsteuer. Mittlerweile liegt ein Gesetzesentwurf der EU-Kommission vor. Auch zur stärkeren Kontrolle des Derivatehandels liegen Gesetzentwürfe vor und europäische Finanzmarktaufsichtsbehörden wurden geschaffen. Dies sind alles Entwicklungen, deren Durchsetzung vor zwei Jahren noch ziemlich unwahrscheinlich erschienen.

Ansatzweise können heute Versuche von Unternehmen der Realwirtschaft beobachtet werden, sich unabhängiger von Banken und Finanzmarkt zu machen. Große Unternehmen wie Siemens, BMW oder Volkswagen haben eigene Banklizenzen, andere wickeln die Finanzierung von Aufträgen oder auch die finanzielle Stützung ihrer Zulieferer verstärkt über eigene Finanzmittel ab.

Das Umdenken zur stärkeren Regulierung sehen wir u.a. als Erfolg des kontinuierlichen Argumentierens von sozialen Bewegungen, NGOs, Gewerkschaften und Teilen des Parteienspektrums. Klar ist aber auch: Dies hat noch nicht dazu geführt, die von den Finanzmärkten ausgehenden kurzfristigen Renditeerwartungen zurückzudrängen. Das ist aber eine notwendige Bedingung, um in vielen anderen Politikfeldern ein nachhaltiges Umsteuern zu ermöglichen. ${ }^{6}$

\subsection{ABBAU DER UNGLEICH- VERTEILUNG VON EINKOMMEN UND VERMÖGEN}

Die in nahezu allen Industrieländern im langfristigen Trend gewachsene Ungleichverteilung von Einkommen und Vermögen ist nicht gebrochen. Die Daten für die Lohn- und Gewinnquoten, die sich in der Krise kurzfristig zugunsten der Lohnquote entwickelt haben, können nicht als Trendumkehr interpretiert werden, da sie primär auf den kurzfristigen Einbrüchen bei Unternehmensgewinnen und Erlösen aus Finanzmarktprodukten beruhen. Diese Effekte konnten die oberen Einkommens- und Vermögensgruppen aber bereits wieder weitgehend kompensieren. Schäfer (in diesem Heft) konstatiert eine „fortgesetzte Polarisierung“ der
Einkommens- und Vermögensverteilung. Dies zeigt sich auch deutlich in den Berechnungen des Deutschen Instituts für Wirtschaftsforschung (DIW), nach denen die Mittelschicht im Zeitraum von 2000 bis 2008 von 66,5 auf $60,9 \%$ deutlich geschrumpft ist - bei gleichzeitigem Wachsen sowohl der Unter- als auch der Oberschicht (DIW 2010). ${ }^{7}$

Der langfristige Trend zur Ungleichverteilung ist eine zentrale Ursache für die Krise. Zwei Effekte greifen ineinander: Die Schwächung der niedrigen und mittleren Einkommen schlägt sich in Ländern wie Deutschland relativ unvermittelt auf die Binnennachfrage nieder. In Ländern wie den USA wurde die schwache Einkommensentwicklung durch ein massives Ansteigen kreditfinanzierten Konsums „kompensiert“. Diese Entwicklung hat auf dem Immobilienmarkt unter anderem die 2007 eskalierte Subprime-Krise verursacht. Die Umverteilung zugunsten der hohen und höchsten Einkommen und Vermögen wirft nicht nur Gerechtigkeitsprobleme auf, sie steigert die Nachfrageschwäche, da diese Gruppen einen großen Teil ihrer zusätzlichen Einkünfte nicht konsumieren, sondern sparen. Diese hohen Sparvermögen fanden dann aufgrund der Nachfrageschwäche keine realwirtschaftlichen Investitionsmöglichkeiten und heizten so die durch politische Deregulierungen ohnehin schon gewachsenen Finanzmarktspekulationen zusätzlich an.

Die wachsende Ungleichverteilung ist aber nicht nur eine Krisenursache, sie macht Gesellschaften für alle Bürger weniger lebenswert: Wilkinson und Pickett haben mit umfangreichem statistischem Material die Auswirkungen von Ungleichheit für Stabilität und Wohlstand von entwickelten Ländern untersucht. Ihre Daten belegen eindeutig, dass mit steigender ungleicher Verteilung von Einkommen und Vermögen auch gesellschaftliche Probleme in vielfältiger Hinsicht zunehmen. Das Spektrum reicht von der Zunahme gesundheitlicher und psychischer Probleme, Kriminalität, abnehmendem Ver-

\footnotetext{
$6 \mathrm{Vgl}$. ausführlicher zum "Problem des Zeithorizontes zentraler Akteure“" Hübner in diesem Heft.

7 Die Schichtgrenzen definiert das DIW bei dieser Berechnung folgendermaßen: Die Unterschicht beginnt bei weniger als $70 \%$ des mittleren Einkommens; die Oberschicht beginnt bei mehr als $150 \%$ des mittleren Einkommens. Entscheidend für unsere Argumentation sind aber nicht die Schichtgrenzen, sondern die Entwicklungen zur Polarisierung.
} 
trauen bis zu steigenden Selbstmordraten (Wilkinson/Pickett 2009). ${ }^{8}$

Wie also sehen die Chancen auf einen Kurswechsel in diesem Politikfeld aus? Welche Akteurskoalitionen bieten sich an?

Für den historischen New Deal lässt sich retrospektiv eine Allianz von langfristig denkenden Kapitalinteressen und den großen Industriegewerkschaften bezüglich der notwendigen Steigerung der Masseneinkommen ausmachen. Eine derartige klassenübergreifende Koalition können wir derzeit noch nicht erkennen. $\mathrm{Zu}$ unübersichtlich sind die Interessenlagen auf der Kapitalseite. Massenkonsumgüter produzierende Unternehmen, die ein eigenes Interesse an stabiler Nachfrage haben müssten, setzen beispielsweise wegen des internationalen Kostenwettbewerbs dennoch auf das Drücken von Lohnkosten. Sie hängen im Zweifelsfall lieber der Illusion nach, dass es auch weiterhin aufstrebende neue Absatzmärkte geben wird. Im gesellschaftlichen Diskurs lassen sich allerdings Verschiebungen der Prioritäten erkennen. Die steigende Ungleichverteilung und ihre negativen Auswirkungen auf den gesellschaftlichen Zusammenhalt werden zumindest wieder in breiteren Kreisen thematisiert - auch bei Menschen am oberen Ende der Einkommens- und Vermögensskala.

\subsection{WELTWIRTSCHAFTLICHE BALANCE}

Die Probleme der gestörten weltwirtschaftlichen Balance zwischen Ländern mit hohen Leistungsbilanzüberschüssen und Ländern mit hohen Leistungsbilanzdefiziten sind sowohl innerhalb Europas als auch global eine Bedrohung für die ökonomische Stabilität. Selbst bei einer auf nationale Grenzen beschränkten Sicht sind für jeden langfristig Denkenden die Risiken dieser Situation auch für Deutschland, das bisher vergleichsweise gut durch die turbulenten Jahre seit 2008 gekommen ist, offensichtlich. ${ }^{9}$ Als exportorientiertes Land sind massive ökonomische Verwerfungen bei unseren Handelspartnern eine Bedrohung der eigenen ökonomischen Prosperität. Ganz zu schweigen von den politischen und sozialen Risiken, die ein Auseinanderbrechen der Eurozone oder weltwirtschaftliche Verwerfungen bedeuten würden.

Der entscheidende Punkt ist aber nicht, dass Deutschland den Pfad einer exportorientierten Industrienation verlassen muss und seine industrielle Stärke quasi demobilisiert. Bei Leistungsbilanzüberschüssen ist immer zu beachten, dass eine Ursache eines starken Exports auch darin liegt, dass er nicht durch entsprechende Importe ausgeglichen wird. Ein geringer Import wiederum hängt eng mit der schwachen Binnennachfrage zusammen. Diese ist unter anderem durch die im Durchschnitt relativ schwache Lohnentwicklung und die gestiegene Ungleichverteilung der Einkommen und Vermögen bedingt (vgl. Lehndorff in diesem Heft).

Hier standen die deutschen Gewerkschaften oft in der Kritik ihrer europäischen Schwestergewerkschaften, die zu niedrige Tarifabschlüsse als Ursache für die deutsche Exportstärke ausmachten. Diese angeblich vorsätzliche Lohnzurückhaltung der deutschen Gewerkschaften wird als unlauterer Wettbewerb interpretiert. Diese Argumentation greift zu kurz. Es handelt sich nicht primär um ein Problem zu niedriger Tarifabschlüsse im deutschen verarbeitenden Gewerbe, in dem die exportorientierten Bereiche der deutschen Wirtschaft beheimatet sind. Hier wurde der verteilungsneutrale Spielraum ausgeschöpft. Vor allem die besonders schwache Einkommensentwicklung in den wachsenden tariflich nicht regulierten Bereichen und die Etablierung eines Niedriglohnsektors wie auch prekärer Beschäftigung sind ursächlich für die vergleichsweise schwache durchschnittliche Einkommensentwicklung in Deutschland (und partiell auch das Absenken übertariflicher Standards).

Festzuhalten ist: Nur mittels Tarifpolitik wird sich die schwache deutsche Einkommensentwicklung und Binnennachfrage nicht stärken lassen. Zum einen ist es eine dringliche Aufgabe für deutsche Gewerkschaften, wieder Einfluss auf die Einkommensbedingungen in den tariffreien Zonen zu bekommen. Zum anderen ist das Zurückdrängen von prekärer Beschäftigung und von Niedriglöhnen ein wichtiger Schritt zu einer gesamtwirtschaftlich stärkeren Einkommensentwicklung. Realistischerweise bedarf es hierzu auch politischer Interventionen in die Arbeitsmarktregulation. Eine weitere wichtige Stellschraube für eine stärkere Binnennachfrage ist die Steuer- und Abgabenpolitik. ${ }^{10}$

Wer sind mögliche Bündnispartner für diese Richtungsänderungen? Ohne in Euphorie zu verfallen und ohne heute wirklich abschätzen zu können, wie viel der Ankündigungspolitik in tatsächliche Politik umgesetzt werden wird, stellen wir dennoch fest, dass die Gewerkschaften nicht mehr die Einzigen sind, die sich gegen prekäre Beschäftigungsverhältnisse und ausufernde Niedriglöhne stemmen. Noch vor einem halben Jahr undenkbar, denkt die CDU über Mindestlöhne nach. SPD und Grüne haben sich in ihrer Programmatik von der Deregulierung des Arbeitsmarktes verabschiedet.

\subsection{BALANCE ZWISCHEN INDUSTRIE UND DIENSTLEISTUNGEN}

Weder der Weg der einseitigen Exportorientierung noch der der kreditfinanzierten Binnennachfrage hat sich als nachhaltige Lösung der seit den 1970er Jahren anhaltenden Wachstums- und Beschäftigungsprobleme der Industrieländer erwiesen.

Greift man den Vergleich der wohlfahrtsstaatlichen Regime auf, so zeigt sich deutlich, dass der skandinavischsozialdemokratische Weg eine Steigerung der Binnennachfrage (und der Arbeitsmarkt- und Verwirklichungschancen der Bürger) auch durch eine deutlich höhere Quote an öffentlich bereitgestellten und finanzierten Humandienstleistungen erreicht. Dies führt wohlgemerkt nicht zu einer Deindustrialisierung dieser Länder, wie dies partiell beispielsweise in Groß-

\footnotetext{
8 Wilkinson/Pickett können natürlich aus ihren Daten keine Aussagen zum „optimalen“ Verhältnis von Ungleichheit und Gleichheit ableiten. Sie können aber eindrücklich belegen, dass die gestiegene Ungleichheit negative gesamtgesellschaftliche Effekte ausgelöst hat und dass alle entwickelten Länder weit vom Wendepunkt entfernt sind, an dem die gesellschaftliche Verteilung in Selbstverwirklichungschancen einschränkender "Gleichmacherei“ umschlägt.

9 Die Betonung liegt auf "vergleichsweise". Allzu oft suggeriert diese Argumentationsfigur, in Deutschland sei ja fast nichts passiert. Unterschlagen wird dabei, dass beispielsweise Instrumente wie die verlängerte Kurzarbeit, die u.a. der international außergewöhnlichen Arbeitsmarktentwicklung in der Hochphase der Krise zugrunde liegen, von den betroffenen Beschäftigten mit erheblichen Einkommensverlusten, also Wohlstandsverlusten "bezahlt" wurden. Auch die langfristigen Belastungen für den Staatshaushalt und die staatliche Handlungsfähigkeit durch die Gegenmaßnahmen sind zu beachten.

10 Schäfer (idH) weist darauf hin, dass es bei der Betrachtung der Steuerpolitik nicht nur um die Lohn- und Einkommensteuer gehen darf, wie sie jetzt wieder von der schwarz-gelben Regierungskoalition aufgegriffen wird. Ein wieder größerer Beitrag der "Gewinnsteuern" zur Finanzierung der öffentlichen Aufgaben sei notwendig.
} 
britannien zu beobachten ist. ${ }^{11}$ Viele der Dienstleistungen - und das zeigt auch die Berufsfeldanalyse - sind mit der Industrie gekoppelt. Die deutlich höheren Investitionen der skandinavischen Staaten in Forschung, Bildung und Erhalt- und Ausbau der beruflichen Qualifikationen über das ganze Arbeitsleben erfüllen auch Anforderungen einer an technologischer Vorreiterschaft und hoher Produktivität orientierten Industrie. Die relativ niedrigen „Dienstleistungswerte“ Deutschlands in diesen Bereichen interpretieren wir als potenzielles Entwicklungshemmnis für die zukünftige industrielle Entwicklung (vgl. hierzu auch Vester in diesem Heft).

Im gesellschaftlichen Diskurs sind Forderungen nach einem Ausbau von Bildung und Forschung, aber auch nach Betreuungsangeboten verankert. In der Frage institutioneller Kinderbetreuungsangebote hat beispielsweise die CDU in den letzten Jahren eine bemerkenswerte Modernisierung ihrer Positionen durchlaufen. Diese Änderungen korrespondieren allerdings nicht mit einem geänderten Verständnis von staatlichen Aufgaben und Finanzierungsnotwendigkeiten und bleiben so für einen tatsächlichen Pfadwechsel relativ wirkungslos.

\section{5 ÖKOLOGISCHER UMBAU DER INDUSTRIEGESELLSCHAFT}

Die Herausforderungen der ökologischen Umgestaltung von Wirtschaftsprozessen werden im Prinzip zwar nur noch von wenigen ganz Unbelehrbaren bestritten. Die Umsetzung einer (ökologisch und sozial) nachhaltigen Industriepolitik ist damit aber noch lange nicht garantiert. Unter den bisherigen kurzfristigen und an betriebswirtschaftlichen bzw. einzelwirtschaftlichen Logiken ausgerichteten Renditemaximierungszielen sind langfristige, ökologisch und sozial nachhaltige Entscheidungen eher unwahrscheinlich.

Eng verbunden mit der Debatte um einen ökologischen Umbau ist die Frage nach wirtschaftlichem Wachstum und der Zukunft der Industrie. Industrie- und Umweltpolitik können nicht getrennt betrachtet werden. Eine industrielle Entwicklung, die auf Raubbau an den natürlichen Lebensgrundlagen der Menschheit beruht, ist nicht zukunftsfähig. Zugleich liegt aber in strikt auf Ressourcen- und Materialeffizienz und Recycling ausgerichteten industriellen Produkten und Produktionsprozessen die Chance für den ökologischen
Kurswechsel. Ökonomisches Wachstum muss vom Naturverbrauch abgekoppelt werden. Wachstumspotenziale müssen in den Feldern erschlossen werden, die zu positiven ökologischen Effekten führen. Ein Orientierungswechsel von blindem Wachstumsglauben zu einem Begriff von qualitativem Wachstum ist notwendig. ${ }^{12}$

Dies würde langfristig zu einer gesellschaftlichen Win-Win-Situation führen. Wie Hübner (in diesem Heft) aufzeigt, gibt es unter dem derzeit herrschenden kurzfristigen Renditedenken keinen Automatismus, der der Win-Win-Logik zum Durchbruch verhilft. Staatliche Interventionen und Investitionsanreize sind nötig. Nicht zuletzt, weil es nicht mit einer Effizienzsteigerung im Umgang mit Ressourcen getan ist, sondern mittelfristig ein grundsätzlicheres Umdenken im Sinne einer Kreislaufwirtschaft nötig ist.

Ein solches staatliches Handeln ist auch geeignet, den Stagnations- und Rezessionsgefahren in Europa zu begegnen. Ein an ökologischen und sozialen Zielen orientiertes europäisches Investitionsprogramm ist notwendig und kann als Neuauflage eines New Deal gedacht werden. Die Forschungs- und Investitionsbedarfe sind enorm. Wenn sie stimuliert werden, kann sich eine wirtschaftliche und ökologische Dynamik entfalten, die einiges Potenzial zur Lösung von Rezessionsgefahren und Unterbeschäftigungsproblemen hat.

In der prinzipiellen Diagnose der notwendigen ökologischen Umsteuerung gibt es breite gesellschaftliche Koalitionsmöglichkeiten. In der Frage einer keynesianisch-inspirierten langfristigen ökologischen und sozialen Investitionsstrategie ist noch einiges an Überzeugungsarbeit zu leisten. Ein Erfolg der gewerkschaftlichen Argumentation der letzten Jahre ist in der Ökologiebewegung selbst auszumachen. Dass der Begriff Nachhaltigkeit nicht auf seine ökologische Dimension reduziert werden kann, sondern auch soziale Nachhaltigkeit mitgedacht werden muss, ist mittlerweile weitgehend Konsens.

\subsection{DEMOKRATISCHE BETEILIGUNG DER BESCHÄFTIGTEN IN DER WIRTSCHAFT}

Die Gewerkschaften verweisen immer wieder zu Recht auf die aktive Rolle, die sie selbst, die Beschäftigten und ihre Betriebsräte bei der Bewältigung der Krise 2008/09 gespielt haben. Sie leiten daraus erweiterte Beteiligungs- und Mitbestimmungsrechte ab.

Schon weit vor Beginn der Krise mussten sich Betriebsräte immer häufiger gegen kurzfristiges Renditedenken zur Wehr setzen. Gegen Kostensenkungs- und Verlagerungsdrohungen wurden vielfach alternative Szenarien entwickelt, ohne einen wirksamen Hebel zu deren Durchsetzung zu haben: Investitionen in Anlagen, in Forschung und Entwicklung, in die berufliche Aus- und Weiterbildung, in die Entwicklung neuer Produkte. Gewerkschaften und die betriebliche Interessenvertretung erwiesen sich dabei nicht selten als engagierte Vertreter einer nachhaltigen Industriepolitik. Um den Forderungen auch Nachdruck $\mathrm{zu}$ verleihen, wird eine echte Mitsprache bei wesentlichen wirtschaftlichen Fragen gefordert. $^{13}$

Argumentiert wird aber nicht nur durchsetzungsorientiert, sondern auch demokratietheoretisch (vgl. Allespach 2011). In der Politikwissenschaft wird inzwischen offen über die aktuellen Gefährdungen der Demokratie gesprochen. Der englische Sozialwissenschaftler Colin Crouch fasst dies in dem Begriff der Postdemokratie zusammen. Nicht mehr der demokratische Souverän, die Wählerinnen und Wähler, sondern die Lobbyisten der Industrie bestimmen zunehmend die Richtlinien der Politik.

Symptome der Demokratiekrise sind: Immer weniger Menschen gehen zur Wahl oder engagieren sich in Parteien. Die Chancen, politisch Einfluss zu nehmen, werden immer geringer eingeschätzt. Die Politikverdrossenheit wächst.

Eine wesentliche Ursache für die allgemeine Resignation und Skepsis ist die

11 Tendenzen bspw. in der schwedischen Politik, den Stellenwert der Industrie gering zu schätzen, was sich beispielsweise in der Krise 2008 in erstaunlich wenigen Maßnahmen zur Sicherung von beruflichen Qualifikationen und Wertschöpfungsketten manifestiert hat, sehen wir als Bruch mit den Leitlinien des skandinavischen Modells. Nicht von ungefähr ist diese wenig aktive Politik von einer liberal-konservativen Regierung verantwortet worden.

12 Ohne dies hier im Detail ausführen zu können, halten wir die Überlegungen der Stiglitz-SenFitoussi-Kommission (vgl. Stiglitz et al. 2009) zu neuen Indikatoren zur Messung von gesellschaftlichem Wohlstand für wichtig und anschlussfähig für die Konzeption eines Begriffs von qualitativem Wachstum. Nur mit dem BIP als Indikator lässt sich das Konzept nicht greifen und nicht statistisch messen.

13 Vgl. hierzu bspw. die Beschlusslage des 22. ordentlichen Gewerkschaftstages der IG Metall im Oktober 2011 
Erfahrung in der Arbeitswelt, dass Menschen wie Objekte herumgestoßen werden. In den Betrieben bedeutet der marktradikale Kurs vielfach:

- die Beschäftigten werden mit unrealistischen Renditeerwartungen gnadenlos unter Druck gesetzt;

- Betriebe werden wegen angeblicher Vorteile ins Ausland verlagert oder die Beschäftigten werden mit der Androhung der Verlagerung erpresst;

- immer mehr Menschen werden in unsicheren Arbeitsverhältnissen beschäftigt;

- Betriebsteile werden abgetrennt, um Lohnkosten zu senken und Mitbestimmung auszuhebeln;

- der Leistungsdruck wird bis zum Anschlag erhöht;

- Betriebe werden aufgekauft und ohne Rücksicht auf die Arbeitsplätze ausgeschlachtet.

Um sich gegen eine derart rücksichtslose Politik wehren zu können, brauchen wir mehr Mitbestimmungs- und Beteiligungsrechte.

Es geht aber zugleich - normativ gewendet - darum, an die Ideengeschichte der europäischen Aufklärung anzuschlieBen. Es geht um die Durchsetzung von Freiheit, Gerechtigkeit und Solidarität. Es geht um ein politisches Projekt, das die Menschen befähigt, ihr Leben selbstbestimmt in die Hand zu nehmen. Es geht um Beteiligung und Mitbestimmung über Themen und Fragen, die die Menschheit gemeinsam angehen. Konkret beinhaltet dies beispielsweise die Erweiterung der betrieblichen Mitbestimmung bei Sanierungskonzepten, bei Betriebsänderungen, bei Beschäftigungssicherung, beim Recht auf externe Beratung, bei Leiharbeit und Werkverträgen, die Herabsetzung der Schwellenwerte für die Unternehmensmitbestimmung, die Aufnahme eines verpflichtenden Katalogs von zustimmungspflichtigen Geschäften in das Aktienrecht. Hierfür gibt es bereits ein Referenzmodell: das VW-Gesetz. Im VW-Konzern können beispielsweise Standortverlagerungen und Massenentlassungen nur mit Zweidrittelmehrheit im Aufsichtsrat getroffen werden, also nicht gegen die Arbeitnehmerbank.

\subsection{NEUE KULTUR DER ARBEIT}

Auf dem Gewerkschaftstag der IG Metall im Oktober 2011 forderte Berthold Huber in sei- nem Zukunftsreferat von der Bundesregierung ein Aktionsprogramm „Neue Kultur der Arbeit“ (vgl. Huber 2011). Ganz im Sinne der Pfaddiskussion formulierte er: „Die Epoche des rücksichtslosen ShareholderKapitalismus ist gescheitert. Wir brauchen eine neue Epoche, in der gute Arbeit die Grundlage unseres Wachstums- und Wohlstandsmodells ist.“ (ebd.) Darin kommt zum Ausdruck, dass das Arbeitsverhältnis für unsere Gesellschaft prägend bleibt.

In Anlehnung an Robert Castel unterscheidet Huber zwischen dem wirtschaftlichen Nutzen und der gesellschaftlichen Anerkennung von Arbeit: „,Der wirtschaftliche Nutzen rechnet sich in Euro und Cent. Die gesellschaftliche Anerkennung drückt sich in sozialen Rechten aus: In Tarifverträgen, fairer Teilhabe, Mitbestimmung und sozialer Sicherung." (ebd.) Marktradikale Politik habe - so Huber weiter - den Zusammenhang von wirtschaftlichem Nutzen, den Arbeit stiftet, und ihrer sozialen Anerkennung aufgelöst. Das belegen zahlreiche Fakten: In Deutschland sind 3 Mio. Menschen offiziell arbeitslos. 7 Mio. Menschen sind gezwungen, für Niedriglöhne auf ungeschützten Arbeitsplätzen zu arbeiten. Prekäre Beschäftigungsverhältnisse nehmen dramatisch zu. Geleistete Arbeitszeit verfällt immer häufiger. Auch der zunehmende Leistungsdruck in den Betrieben zeigt, wie wirtschaftlicher Nutzen und die soziale Anerkennung von Arbeit auseinanderdriften. Arbeitsbeziehungen werden zunehmend vermarktlicht. Jeder Beschäftigte soll als Individuum konkurrenzfähig sein, muss seine Rentabilität täglich unter Beweis stellen. Wer sich nicht "rechnet“, dem drohe die Ausgrenzung, bis hin zur Arbeitslosigkeit (vgl. ebd.). Um in Abgrenzung zu solchen Entwicklungen den Zusammenhang zwischen Anerkennung und Nutzen von Arbeit wieder herzustellen, sind Aktivitäten auf vielen Handlungsfeldern gefordert: In der Betriebspolitik, der Tarifpolitik und nicht zuletzt in der Gesellschaftspolitik.

Das von Huber geforderte Aktionsprogramm greift eine Initiative zur Umsetzung des Beschlusses des Bundeskabinetts von Meseberg zur Humanisierung der Arbeit auf. Es schließt zugleich an das große gesellschaftliche Projekt „Humanisierung der Arbeitswelt" aus den 1970er Jahren an. Der Begriff „neue Kultur der Arbeit“ spannt dabei den Bogen weit: Es geht um Arbeits- und Gesundheitsschutz, um Arbeitsgestaltung und Produktionssysteme, um Beteiligungsorientierung, um eine Arbeitszeitpolitik, die neben einer erhöhten Flexibilität für die Betriebe auch zu mehr Zeitsouveränität für die Beschäftigten führt, es geht um alters- und alternsgerechte Arbeitsbedingungen, um umfassende berufliche Aus- und Weiterbildung, um nur einige Aspekte zu nennen. Es geht dabei aber auch um Nachhaltigkeit und den Ausbau arbeitsorientierter Forschung.

\subsection{HANDLUNGSFÄHIGER, EINNAHMESTARKER STAAT}

Bund, Länder und Gemeinden hätten im Jahr 2011 über 51 Mrd. $€$ mehr an Steuern eingenommen, wenn noch die Steuergesetze von 1998 gälten (IMK 2011). Der von uns skizzierte Kurswechsel kommt ohne einen handlungsfähigen und einnahmestarken Staat nicht aus. Die Erosion der staatlichen Einnahmen ist im Übrigen auch ein oft unterbelichteter Aspekt wachsender Ungleichverteilung, der Umverteilung zwischen öffentlichen und privaten Mitteln. Entgegen weit verbreiteten Vorurteilen sind die öffentlichen Ausgaben in Deutschland von 1998 bis 2008 preisbereinigt im Mittel um 0,2 \% gesunken (ebd.). 2007 und 2008 erreichte die Staatsquote mit 43,6 bzw. 43,8\% die niedrigsten Werte seit 1990 (BMF 2011). Dies ist eine verdeckte Form der Umverteilung, da in der Regel die Bezieher niedriger Einkommen nicht auf marktliche Angebote ausweichen können. Von einer eingeschränkten oder in ihrer Qualität abgesunkenen Versorgung mit öffentlichen Gütern (z.B. Bildung) werden so besonders die Teilhabechancen der mittleren und niedrigen Einkommensgruppen negativ beeinflusst. Es geht also nicht nur um die Verteilung von Einkommen und Vermögen, sondern auch um die Verteilung von Lebens- und Verwirklichungschancen.

In Zusammenhang mit den Diskussionen um den bröckelnden gesellschaftlichen Zusammenhalt wird auch die Einnahmeseite des Staates aufgegriffen. Es ist bemerkenswert, dass die derzeitige Regierung mit ihren Steuersenkungsplänen eher Wähler verschreckt als gewonnen hat. Selbst einige Reiche fordern, höher besteuert zu werden, damit der Staat seinen Aufgaben nachkommen kann. Der Diskurs wird aber relativ defensiv, mit dem Schwerpunkt auf der Verschuldungsproblematik und nicht im Sinne eines aktiven in die Verwirklichungschancen seiner Bürger investierenden Staates, geführt. „Die Steuerreformen 
der rot-grünen Regierung waren ein kapitaler strategischer Fehler und führten zur Befestigung eines neoliberalen Pfades der fahrlässigen Autonomieeinschränkung sozialdemokratischer Politik der Zukunft" konstatiert der Politikwissenschaftler Wolfgang Merkel (Merkel 2010). Der politische Wille, diesen eingeschlagenen Pfad zu verlassen, ist bei der SPD und den Grünen bisher nur partiell zu erkennen. Analog zur effektiven Regulation der Finanzmärkte, wäre ein solcher Richtungswechsel aber eine notwendige Bedingung für einen Kurswechsel und eine nachhaltige Bekämpfung der Krisenursachen.

\section{Schlussfolgerungen}

Weder aus der Pfaddebatte noch aus unserer Studie zur Entwicklung der Berufsstruktur (s.o.) kann eine Blaupause für eine neue gesellschaftliche Balance abgeleitet werden. Die eine funktional richtige, von allen gesellschaftlichen Kräften geteilte Antwort gibt es nicht. Eine solche Vorstellung wäre - bei allen aufgezeigten Interessensübereinstimmungen - eine ahistorische, sozialtechnische Illusion.

Weder die Hoffnung auf einen Masterplan noch auf die allein seligmachende krisenüberwindende Einzelmaßnahme sind realistisch. Das ist aber auch nicht unsere Erwartung an die Pfaddebatte und die Analyse der Berufsstruktur. Dafür sind die Feldzusammenhänge zu komplex.

Die Pfaddebatte sehen wir als ein Instrument der Identifikation gesellschaftlicher Pfade, Pfadabhängigkeiten und Pfadwechselgelegenheiten. Die Rolle von Gewerkschaften lässt sich dabei nicht auf die wissenschaftliche Analyse reduzieren. Gewerkschaften sind aktive politische Akteure und Teile von Interessenkoalitionen und Interessengegensätzen. Aus gewerkschaftlicher Perspektive geht es auch darum, auf Pfadwechselgelegenheiten und sie tragende Akteurskonstellationen im Sinne eines Agenda-Setting aktiv hinzuarbeiten. Gewerkschaften können aus der Pfaddebatte wichtige Informationen für ihre eigenen Handlungsoptionen und Strategien ziehen.

Die Analyse der Berufsstruktur ist ein Instrument, um die Auswirkungen gesellschaftlicher Pfade auf die Berufsstruktur differenziert zu identifizieren. Die Ergebnisse sind geeignet, die in den nationalen
Diskursen weitverbreitete Annahmen, z.B. zur Ingenieursdichte, vergleichend zu hinterfragen.

Sehr konkrete Schlussfolgerungen für ihre Mitgliederpotenziale und Politikschwerpunkte können Gewerkschaften aus den Verschiebungen der Qualifikationsstrukturen ablesen. Es lassen sich Prognosen über die zahlenmäßige Entwicklung von Beschäftigtengruppen ableiten. Für die Gewerkschaften zeichnet sich eine Bedeutungszunahme von höher qualifizierten Beschäftigten ab. Aber diese Entwicklung ist in Deutschland vergleichsweise gebremst. Ob sich diese Dynamik beschleunigt oder weiter abbremst, ist nicht quasi naturwüchsig durch die ökonomische Entwicklung determiniert. Gesellschaftspolitische Entscheidungen machen den Unterschied.

Unabhängig von der Intensität dieser Dynamik ergeben sich deutliche Gestaltungsanforderungen für Gewerkschaften. Für die angestammte Kernklientel der IG Metall, die Facharbeit, müssen erweiterte Strategien der beruflichen Entwicklung im Sinne von Aufstiegs- und Erhaltungsqualifikationen durch Weiterbildung und eine größere Durchlässigkeit zwischen den beruflichen Entwicklungswegen der dualen Ausbildung und akademischer Abschlüsse etabliert und durchgesetzt werden. Die deutschen Industriegewerkschaften haben auf diese Herausforderungen bereits reagiert: z.B. mit tarifvertraglichen Regelungen zur Weiterbildung. Wir möchten aber festhalten: Gerade in Deutschland ist Facharbeit kein Auslaufmodell. Vielmehr müssen Facharbeit und duale Ausbildung stärker mit anderen Qualifikationswegen vernetzt - und nicht durch sie ersetzt - werden.

Ein übergeordnetes Ziel des einzuschlagenden Entwicklungsweges ist es, die Emanzipation und Mündigkeit sowie die Verwirklichungschancen der Bürger zu erhöhen. Der skandinavische Wohlfahrtsstaat ist in diesem Zusammenhang nach wie vor ein wichtiges Referenzmodell für gewerkschaftliche (und sozialdemokratische) Zielsetzungen - nicht so sehr in der konkretistischen Übertragung von Ausgestaltungen wie z.B. der stärkeren Steuerfinanzierung der sozialen Sicherung, ${ }^{14}$ sondern in den Zielsetzungen: geringere Ungleichheit, relativ große gesellschaftliche Integration, größere Gleichheit bei den Arbeitsmarktchancen zwischen den Geschlechtern, individuelle Bildungs- und Verwirklichungschancen für alle, ein zu- kunftsfähiger Arbeitsmarkt, ein investiver und absichernder Wohlfahrtsstaat usw.

Für einen Kurswechsel brauchen Gewerkschaften eine politische Strategie, die einerseits langfristige Ziele formuliert. Andererseits muss sie Gewerkschaften im Hier und Jetzt handlungsfähig halten. Unter Bezug auf den französischen Soziologen Robert Castel lässt sich eine solche Strategie als linker Reformismus bezeichnen (Castel 2010, S. 219ff.). Ein linker Reformismus sucht immer wieder neu nach Wegen für die Emanzipation der Menschen. In möglichst aufeinander abgestimmten Schritten wird ein gesellschaftlicher Pfadwechsel angestrebt. Realistischerweise muss eine solche Strategie dennoch offen sein für das pragmatische Aufgreifen sich anbietender Handlungsspielräume und Koalitionen. Er passt sich nicht an, sondern will eingreifen, gestalten und verändern. Im Ringen um eine gerechtere Gesellschaft ist ein linker Reformismus unseres Erachtens die fortschrittlichste und realistischste Option. Die Meßlatte für den Erfolg eines linken Reformismus sind die Bedingungen für ein selbstbestimmtes, gutes und erfülltes Leben.

Die Frage, wer die Träger eines neuen gesellschaftspolitischen Leitbildes sein werden, lässt sich heute noch nicht im Einzelnen beantworten. Wir haben exemplarisch Hinweise gegeben. Wichtig ist, abschließend festzuhalten, dass innerhalb des sogenannten bürgerlichen Lagers Fraktionen erstarken, die neoliberale Prioritäten ersetzen möchten durch Konzepte einer langfristigen Konversion, die die gestörte wirtschaftliche und soziale Balance durch die Erweiterung der Human- und Sozialdienstleistungen und der Bildungschancen, ein moderneres Familienmodell und eine (profitable) ökologische Industriepolitik ein Stück weit korrigiert. Ihr Interesse liegt natürlich nicht darin, die soziale Hierarchie, in der sie die Hegemonie haben, anzutasten. Es ist Aufgabe einer gewerkschaftlichen Strategie, die gesellschaftlichen Aushandlungsprozesse, die der Suche und den Kompromissen einer neuen gesellschaftlichen Balance vorausgehen, zu nutzen, um ein Stück weit die soziale Hierarchie und die wirtschaftlichen Machtverhältnisse zu verändern. Das ist kein Automatismus, aber eine reelle Chance.

14 Vgl. zur Frage von Übertragbarkeit bzw. notwendigen Adaption von Strukturmerkmalen zwischen den verschiedenen wohlfahrtsstaatlichen Typen auch Bogedan (in diesem Heft). 


\section{LITERATUR}

Allespach, M. (2011): Kurswechsel! Warum mehr Mitbestimmung und Wirtschaftsdemokratie die Marktwirtschaft gerechter und stabiler machen, Vortrag Kreisky-Forum Wien, 12. Mai (unveröffentlicht) Bundesministerium der Finanzen (BMF) (2011): Entwicklung der Staatsquote, http://www.bundesfinanzministerium.de/nn_4316/DE/ BMF__Startseite/Service/Downloads/Abt__I/Entwicklung__der__Staat squote__26012011, templateld =raw, property= publicationFile.pdf Castel, R. (2011): Die Krise der Arbeit. Neue Unsicherheiten und die Zukunft des Individuums, Hamburg

Esping-Andersen, G. (1990): The Three Worlds of Welfare Capitalism, Cambridge

Esping-Andersen, G. (Hrsg.) (1993): Changing Classes. Stratification and Mobility in Post-Industrial Societies, London

Esping-Andersen, G. (1998): Die drei Welten des Wohlfahrtskapitalismus. Zur Politischen Ökonomie des Wohlfahrtsstaates, in: Lessenich,

St./Ostner, I. (Hrsg.): Welten des Wohlfahrtskapitalismus,

Frankfurt a. M., S. 16-56

Esping-Andersen, G./Gallie, D./Hemerijck, A./Myles, J. (2002): Why We Need a New Welfare State, Oxford

Fitoussi, J.-P./Stiglitz, J. (2009): The Ways Out of the Crisis and the Building of a more Cohesive World, Document de travail OFCE (Observatoire Francais des Conjonctures Économiques) 2009-17, http://www. ofce.sciences-po.fr/pdf/dtravail/WP2009-17.pdf

Galbraith, J. K. (1967): The New Industrial State, London

Goebel, J./Gornig, M./Häußermann, H. (2010): Polarisierung der Einkommen. Die Mittelschicht verliert, in: DIW-Wochenbericht 77 (24), S. $2-8$

Groh-Samberg, O. (2007): Armut in Deutschland verfestigt sich, in: DIW-Wochenbericht 74 (12), S. 177-182

Groh-Samberg, O. (2009): Armut, soziale Ausgrenzung und Klassenstruktur, Wiesbaden

Huber, B. (2010): Kurswechsel für Deutschland. Die Lehren aus der Krise, Frankfurt/New York
Huber, B. (2011): Zukunftsreferat auf dem 22. ordentlichen Gewerkschaftstag der IG Metall am 12. Oktober 2011 in Karlsruhe, http:// www.igmetall.de/cps/rde/xbcr/internet/11_10_12_\%20Zukunftsreferat BertholdHuber_Journalisten_0178977.pdf

IG Metall (2011): Karlsruher Erklärung, Initiativantrag zur Entschließung 1, http://www.igmetall.de/cps/rde/xbcr/internet/docs_ig_metall_ xcms_179073_179074_2.pdf

Institut für Makroökonomie und Konjunkturforschung (IMK) (2011): Staatsausgaben trotz Krisenabwehr real kaum gestiegen. In diesem Jahr 51 Milliarden Euro Mindereinnahmen durch Steuersenkungen, Pressemitteilung vom 08.09., http://www.boeckler.de/6936_37820.htm Kohl, J. (1993): Der Wohlfahrtsstaat in vergleichender Perspektive. Anmerkungen zu Esping-Andersens "The three worlds of welfare capitalism“", in: Zeitschrift für Sozialreform 39 (2), S. 67-82

Marshall, Th. H. (1989) [1950]: Citizenship and Social Class, Cambridge Merkel, W. (2010): Falsche Pfade? Probleme sozialdemokratischer Reformpolitik, in: Neue Gesellschaft/Frankfurter Hefte (7/8), S. 72-75 Mills, C. W. (1948): The New Men of Power - America's Labor Leaders, New York

Müller, W. (1998): Erwartete und unerwartete Folgen der Bildungsexpansion, in: Kölner Zeitschrift für Soziologie und Sozialpsychologie (KZfSS) 50 (Sonderheft 38), S. 81-112

Schirrmacher, F. (2011): Bürgerliche Werte "Ich beginne zu glauben, dass die Linke recht hat" ", in: Frankfurter Allgemeine (FAZ) vom 14.08 Stiglitz, J./Sen, A./Fitioussi, J.-P. (2009): Report by the Commission on the Measurement of Economic Performance and Social Progress, http://www.stiglitz-sen-fitoussi.fr/documents/rapport_anglais.pdf Vester, M./Gardemin, D./Groh-Samberg, O. (2009): Zwischenergebnisse der Berufsfeldanalyse nach Oesch auf der Grundlage des Sozioökonomischen Panels (SOEP) 1990-2007, Forschungsbericht, Hannover Wilkinson, R./Pikett, K. (2009): Gleichheit ist Glück. Warum gerechte Gesellschaften für alle besser sind, Berlin 